\title{
THE EFFECTS OF SELECTION AND HUMAN PREFERENCE ON COAT COLOUR GENE FREQUENCIES IN URBAN CATS
}

\author{
J. M. CLARK \\ Institute of Genetics, University of Glasgow, Glasgow, G11 5JS, Scotiand
}

Received 22.i.75

\begin{abstract}
SUMMARY
In an attempt to examine the possibility of selection on domestic cat populations in the urban environment, a number of surveys were conducted in four contrasting areas of Glasgow - two near either extreme of Western, industrial socioeconomic status. The first survey was in the form of a questionnaire to inhabitants enquiring of preference for a particular phenotype. This survey revealed a consistent preference for lighter phenotypes in all areas. The incidence of cat ownership was greatest in the two affluent areas where there was also a high risk of the cat being sterilised.

Independent of the questionnaire survey was a study of cat coat colour gene frequencies in each of the four districts. This study revealed that darker phenotypes predominate in the two areas of lower socioeconomic status, Maryhill and Govan. To date, the cat populations in these two areas are the darkest to have been described in the world. In contrast, a lighter phenotype was found in cats inhabiting the two districts of higher socioeconomic status, Kelvinside and Bellahouston. This difference in phenotypic darkness was also paralleled by appropriate clines in coat colour gene frequencies. The results demonstrate that human preference is not effectively translated into selection at any of the loci considered. In situations where human preference is most likely to occur, neutering risk is also high.

Although the force responsible for the darkening of phenotype has yet to be identified, the fact that the darker alternative allele at each of the $O, a, t$, $d, S$ and $W$ loci is favoured suggests that selection is operating on these polymorphic coat colour loci.
\end{abstract}

\section{INTRODUCTION}

THE increasing amount of data on cat coat colour gene frequencies invite discussion in terms of evolutionary processes such as mutation, natural and artificial selection, gene flow and genetic drift. As a result of human preference the domestic cat may not be an ideal organism with which to study the genetics of populations. However, it is rare that we can study a mammalian population and so readily score phenotypes for a number of polymorphic loci. Most, if not all, studies to date have shown urban populations of Felis catus to be panmictic. For these reasons alone it seems worth while persevering with the problem and elucidating the extent and effects of human preference for certain phenotypes. We may then be able to assess likely contributions of cat population genetics to fundamental problems of evolution.

A number of authors have suggested the possible importance of human preference in the interpretation of cat coat colour gene frequencies. Searle (1949) states that the polymorphic cat population of London was maintained as such by human selection-this selection being mainly exercised on litters rather than on choice of mate. Dreux (1967), Robinson and Silson (1969), 
Robinson (1972) and Dreux and Legel (1973) have all suggested that human preference may be an important, if not indeterminate agent of artificial selection. In a study of cats in York, Metcalfe and Turner (1971) observed that selection occurred at the orange $(O)$ locus and suggest it may be the result of human preference. These authors maintain that artificial selection by human preference takes place in circumstances probably accessible only by the methods of sociology. Clark (1973) suggests that cat gene frequencies in Australian cities may be influenced by different human preference patterns.

The surveys described in this paper were conducted as an attempt to document the effects of human preference on at least one cat population and to examine whether preference leads to gene frequency differences within this population. Attention was also paid to the problem of distinguishing between natural and artificial selection.

\section{Methods \\ (i) Human preference survey}

In order to assess the effects of human preference on cat populations, a survey was conducted in four old, inner areas of Glasgow, Scotland. These areas were Kelvinside, Bellahouston, Maryhill and Govan (see fig. 1). The first two areas are mainly inhabited by persons in socioeconomic groups 1, 2, 3, 4 whilst the latter two areas are mainly inhabited by persons in groups 5 and 6 . The inhabitants of all four areas were predominantly of British origin. The socioeconomic classification is in accord with the scale used by Cavalli-Sforza and Bodmer (1971), where groups 1 to 4 represent those in professional, clerical and skilled occupations, and groups

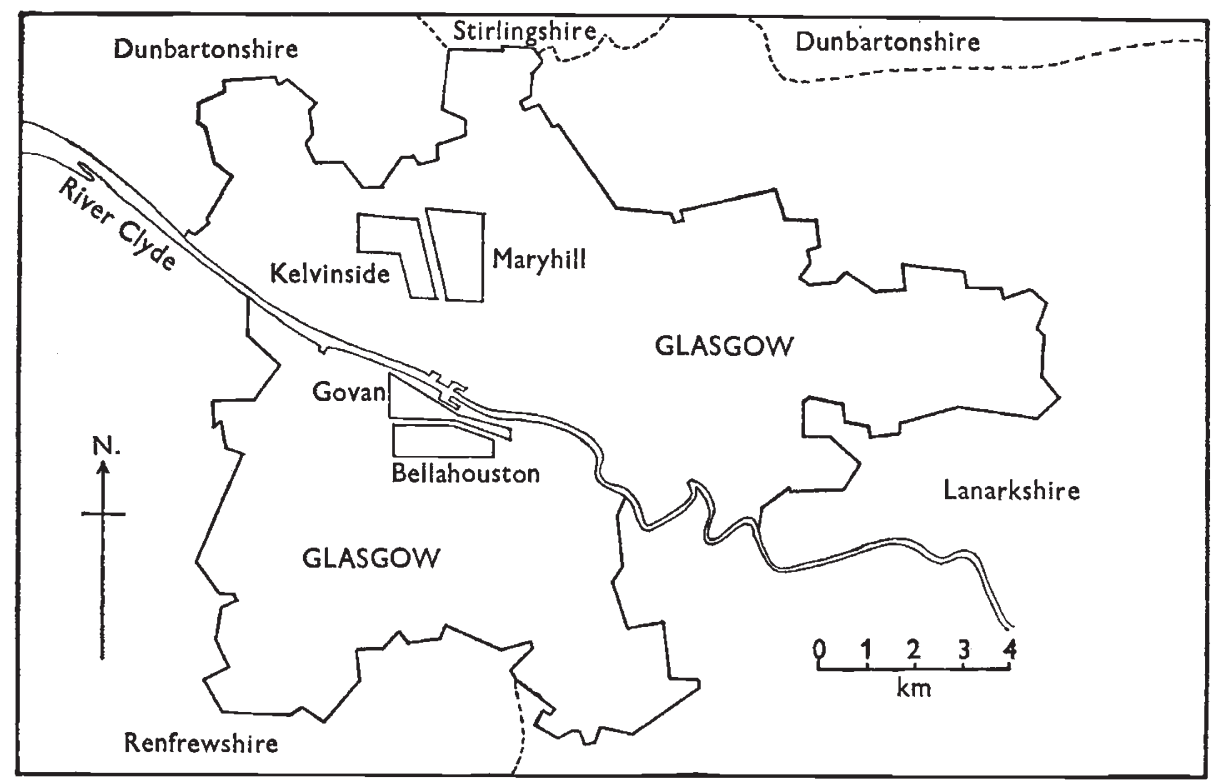

FrG. 1.-The four regions in Glasgow selected for a study of human preference for certain cat phenotypes and a survey of cat coat colour gene frequencies. 
5 and 6 those who are semi-skilled and unskilled. These areas were selected so as to represent two geographically separated samples near both extremes of Western, industrial, human socioeconomic status. Kelvinside and Bellahouston, which certainly appear more affluent than Maryhill and Govan, are characterised by quiet suburban streets, detached dwellings and private gardens. In contrast, Maryhill and Govan contain large tenement blocks, many derelict buildings and are undergoing a considerable amount of redevelopment.

The method of assessing human preference was to distribute a questionnaire amongst households selected at random. The individuals were asked to fill out the form with minimum assistance from the examiner. One form was completed per household. The 7 per cent of individuals who showed a strong dislike of cats and failed to answer the questionnaire were excluded from the sample. This will result in a slight overestimate of cat ownership. There was no difference in the rate of refusal between the four areas.

The survey form was written so as to enquire of preference for a certain coat colour, coat length and sex; superstitious beliefs with respect to black cats; ownership of cat at present and within the last five years; the origin of these cats; influence of children on selection of the cat; a description of these animals and the causes of death, if any. Only people who had lived in the area for five years or more were considered in the sample. Cats from outside Glasgow were excluded. The survey specifically excluded Siamese cats and "fancy" cats kept for pedigree or show purposes. Litters and known siblings were rarely encountered and not included in the survey.

\section{(ii) Cat survey}

Independent of the human preference survey was a survey of both owned and stray animals from the four areas described above. Observations were made on the following allele pairs: sex-linked non-orange, orange $\left(0^{+}\right.$, O) and autosomal loci controlling agouti, non-agouti $\left(a^{+}, a\right)$, striped and blotched tabby $\left(t^{+}, t^{b}\right)$, non-dilute, dilute $\left(d^{+}, d\right)$, short hair, long hair $\left(l^{+}\right.$, $l)$, dominant piebald spotting, non-spotted $\left(S, s^{+}\right)$, dominant white, pigmented $\left(W, w^{+}\right)$. The genetics of these mutants is described in Robinson (1959). The degree of white spotting was determined using the scale suggested by Dreux (1967) and Dreux and Legel (1973), however, restricted white spotting was not assessed. Litters and siblings were not included. With the exception of $O$, gene frequency estimates were found as the square roots of the appropriate phenotype proportions. Variances of all estimates are described by the negative reciprocal of the second derivative of the log likelihood function (Cook, 1971). The standard error of these estimates is taken as the square root of the variance.

\section{Results}

No elaborate statistical treatment of the data has been performed and the problem of sampling error must be kept in mind. Where appropriate, a chi-square test has been employed and generally represented as the maximum or minimum value for differences between areas considered. The appendix contains detailed results of the questionnaire survey. A summary of human preferences in the four areas can be found in table 1 


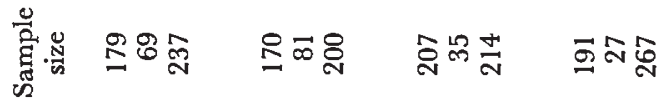

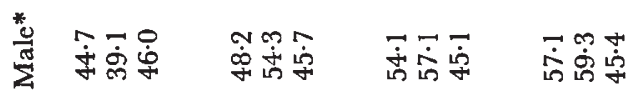

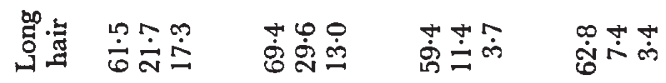

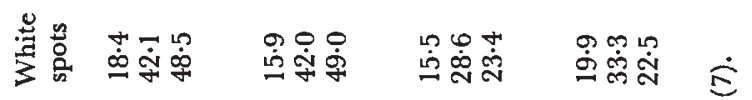

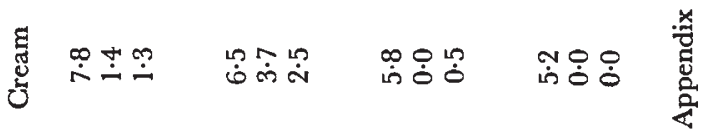

弟

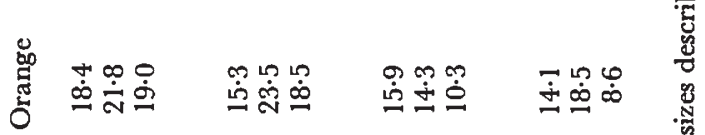

嵌

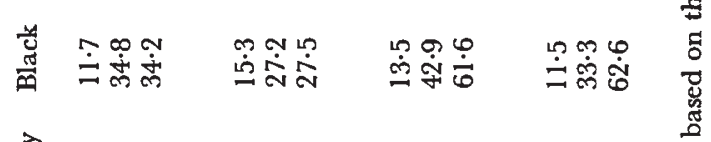

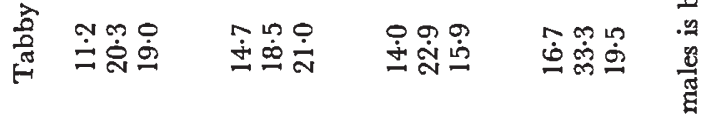

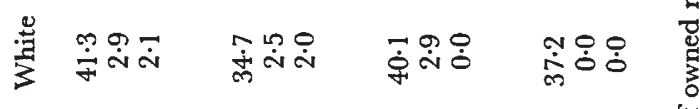

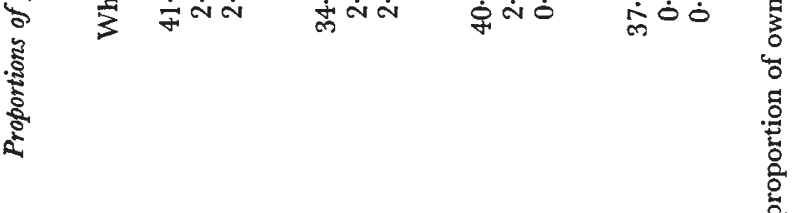

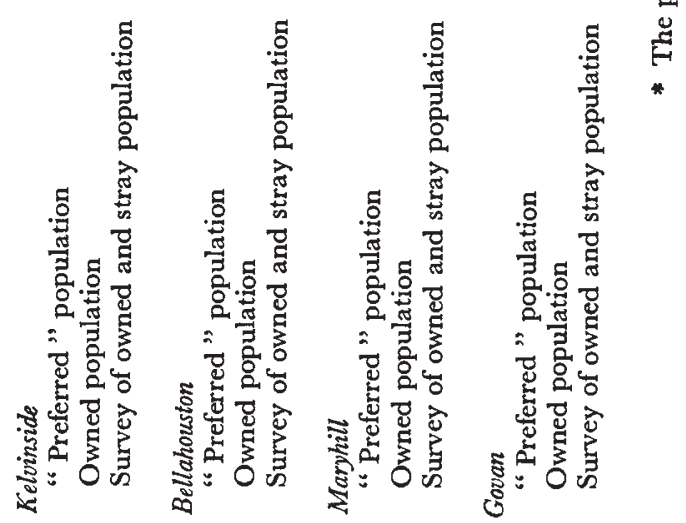


and will be considered in more detail below. The questionnaire results suggest that there is little difference in human preference patterns between the four areas. The only obvious difference is a greater preference for male cats in Maryhill and Govan than in Kelvinside and Bellahouston ( $\left.\chi^{2}>5 \cdot 67\right)$. This means that the minimum $2 \times 2$ contingency chi-squared value between the two types of area is greater than $5 \cdot 67$. An explanation for this could lie in the problems associated with female cats and kittens. The questionnaire sample size is inadequate for the purpose of detecting differences in human preference and greater care in the presentation of the questionnaire would probably be necessary to investigate this problem. However, for the purpose at hand a few general observations can be made from the results of the questionnaire.

Superstition may be an important factor when considering the fate of black cats. Appendix (2) shows that 16-26 per cent of the people interviewed held superstitious beliefs about black cats. However, the data show a similar proportion of people regarding black cats as being either lucky or unlucky. This would suggest that any preference effects attributed to superstition may tend to cancel each other.

There is a notable difference in the frequency of cat ownership between the four areas considered (see Appendix (4)). Cat ownership is expressed in terms of households rather than individuals. The data show that cat ownership is least in the less affluent areas of Maryhill and Govan. This suggests that humans in these areas are likely to have less opportunity to express their preference for the coat colours considered. In contrast, the incidence of ownership in Bellahouston (approximately 40 per cent) indicates that individuals in this region may have a greater opportunity to influence the cat population. However, Appendix (7) shows that the proportion of neutered animals is greater in the more affluent Kelvinside and Bellahouston areas.

The majority of owned cats were either adopted "strays" or " gifts" to the household. A relatively small percentage of animals were obtained from pet shops or welfare homes (see Appendix (5)). Interestingly, a large proportion of pets came from outside the district considered. The fact that perhaps one-half of the owned animals were immigrants to the district suggests that there may be considerable opportunity for gene flow between the city districts. This gene flow would be more significant in Kelvinside and Bellahouston where the incidence of ownership is greatest. As a result of the low incidence of cat ownership, human-assisted gene flow into Maryhill and Govan is expected to be less significant.

The influence of children on the selection of a cat is shown in Appendix (6). Children in the two more affluent areas exert a greater influence in the choice of an animal. Upon further questioning of the respondents, it was evident that white spotting was the most common phenotype preferred by children. Todd (personal communication) has previously suggested that the frequency of $S$ may be increased by child preference for this phenotype. Presumably piebald spotting may provide a distinctive recognition clue.

Although the numbers are limited, and classification unsatisfactory, Appendix (8) provides interesting information on the cause of death of cats kept in the four areas over the last five years. The cause of death was recorded in three vague categories. Cats which "disappeared" were not 
considered. Attempts were made to ensure that death from illness was the result of a pathological condition rather than an accident. Non-accidental death of animals greater than 7 years old (an arbitrary limit) was described as the result of "old age". Accidental death is the most prominent cause in Maryhill and Govan. Deaths mainly resulted from automobiles or delinquency (" cat bashing" is a common pastime in these areas). Illness was also a significant cause of death. In contrast, animals in Kelvinside

TABLE 2

Orange locus phenotypes, sex-ratio and $\mathrm{O}$ frequency in sexed animals

$\begin{array}{lcccc} & \text { Kelvinside } & \text { Bellahouston } & \text { Maryhill } & \text { Govan } \\ \begin{array}{l}\text { Phenotype } \\ +/+\end{array} & 49 & 55 & 41 & 66 \\ + \text { IO } & 42 & 29 & 7 & 9 \\ \text { O/O } & 11 & 10 & 2 & 2 \\ +/- & 59 & 54 & 35 & 57 \\ \quad \text { O/ } & 28 & 25 & 6 & 7 \\ \begin{array}{l}\text { Deviation from expecta- } \\ \text { tion } \chi^{2}\end{array} & 0.21 & 4.38 & 3.84 & 4.28 \\ \begin{array}{l}\text { Male/female sex ratio } \\ \text { Frequency of } O \text { based on } \\ \text { sexed animals }\end{array} & 0.853 & 0.840 & 0.820 & 0.831 \\ & 0.316 \pm 0.034 & 0.277 \pm 0.034 & 0.121 \pm 0.034 & 0.092 \pm 0.024\end{array}$

and Bellahouston appear to be less at risk and old age becomes the major cause of death. Details of the cats kept by respondents in the last five years appear in Appendix 9 and Table 1.

Independent of the human preference questionnaire and survey of cats owned by respondents to the questionnaire, was a study of the cats in each area. The sample was obtained by door-to-door canvass and casual observations. Where possible, sex was recorded. Randomness of mating was determined, as in previous studies, by a comparison of theoretical and observed frequencies of black, tortoiseshell and orange phenotype frequencies. The results for sexed animals in table 2 show that for all samples, deviation from expectation was small, and hence the four populations are regarded as panmictic. Male/female sex-ratios were similar in all four areas. The frequency of $O$ is calculated in two ways. In table 2 appear estimates of $O$ based on only the sexed animals, where five classes are recognised. The values for $O$ which appear in table 3 are maximum likelihood estimates (Robinson, 1972) based on all the animals scored for this locus. Differences between the two estimates are small and the errors overlap to a considerable extent.

For the purposes of comparison with samples obtained from the questionnaire, proportions of phenotypes in the cat survey are presented in table 1 . Comparison of the three "populations" will be made below. Gene frequency estimates for all seven loci can be found in table 3. These data show that cat populations in areas with similar environments (both physical and human) also show similar gene frequencies. Thus Kelvinside and Bellahouston are similar, but both different from Maryhill and Govan. Significance tests reveal that the least difference between the two environments is still significant for all loci (orange, $\chi^{2}>7 \cdot 25$; non-agouti, $\chi^{2}>8 \cdot 10$; blotched tabby, $\chi^{2}>8 \cdot 79$; dilute, $\chi^{2}>7 \cdot 83$; long hair, $\chi^{2}>11 \cdot 76$; white spotting, 
SELECTION IN URBAN GATS

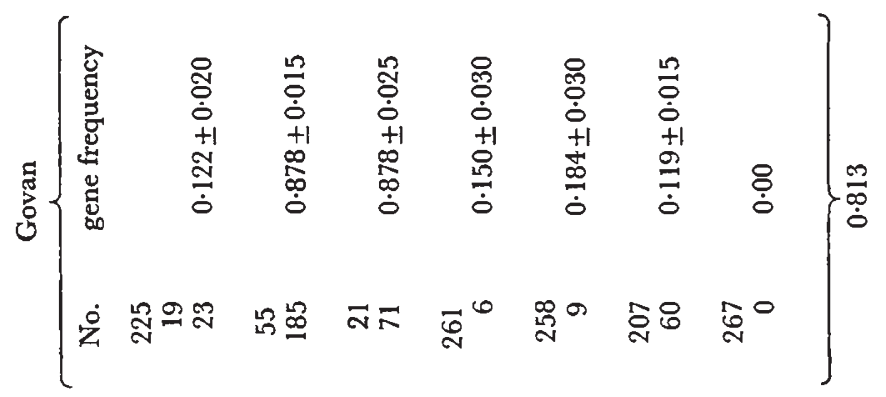

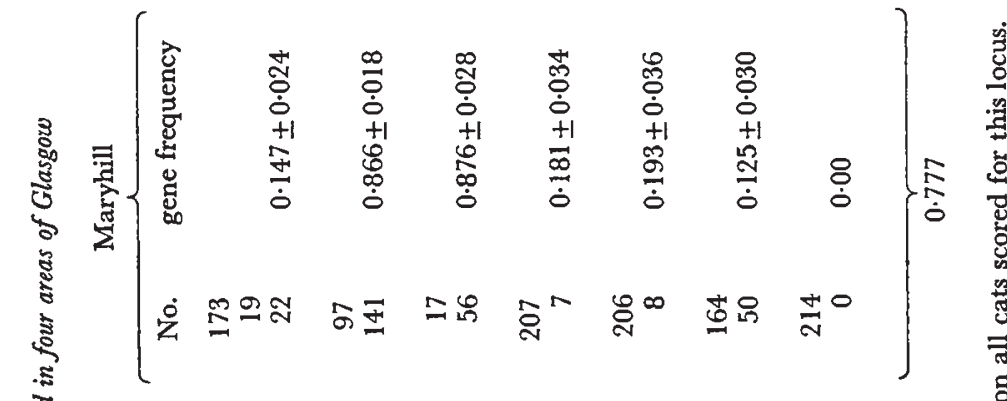

क है

है

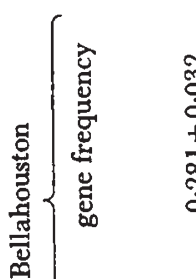

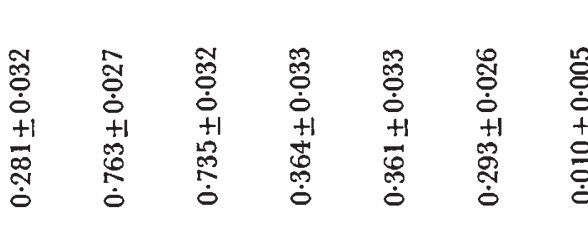

(1)

ํำ

$\circ]$

๕ัญ

(⿻)

ธุ

कू

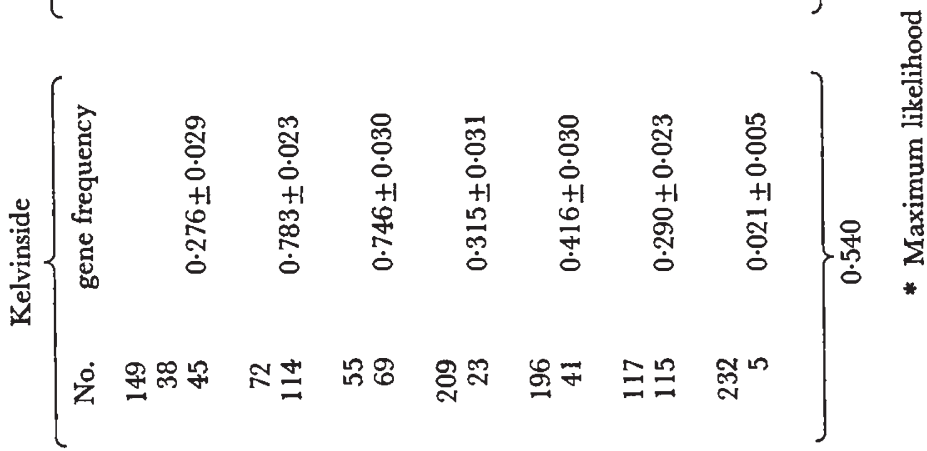

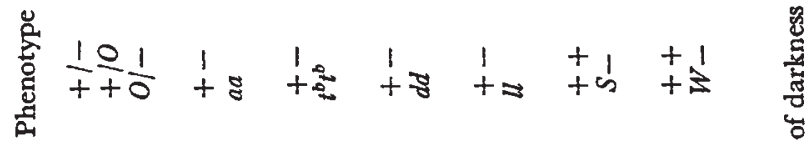

:

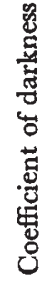


$\chi^{2}>32.79$ ). There is a conspicuous trend in these data. The darker alternative of the allele pair is more frequent in Maryhill and Govan. This apparent shift towards a darker phenotype can also be shown by comparing coefficients of darkness which appear in table 3 . The coefficient of darkness described by Todd (1969) is the frequency for males of the two darkest phenotypes controlled by alternative alleles at the loci $O, a, t, d$. For comparison, the gene frequencies of cats in York are: $O, 0.20 ; a, 0.81 ; t^{b}$, $0.78 ; d, 0.27$ (Metcalfe and Turner, 1971). This sample represents the

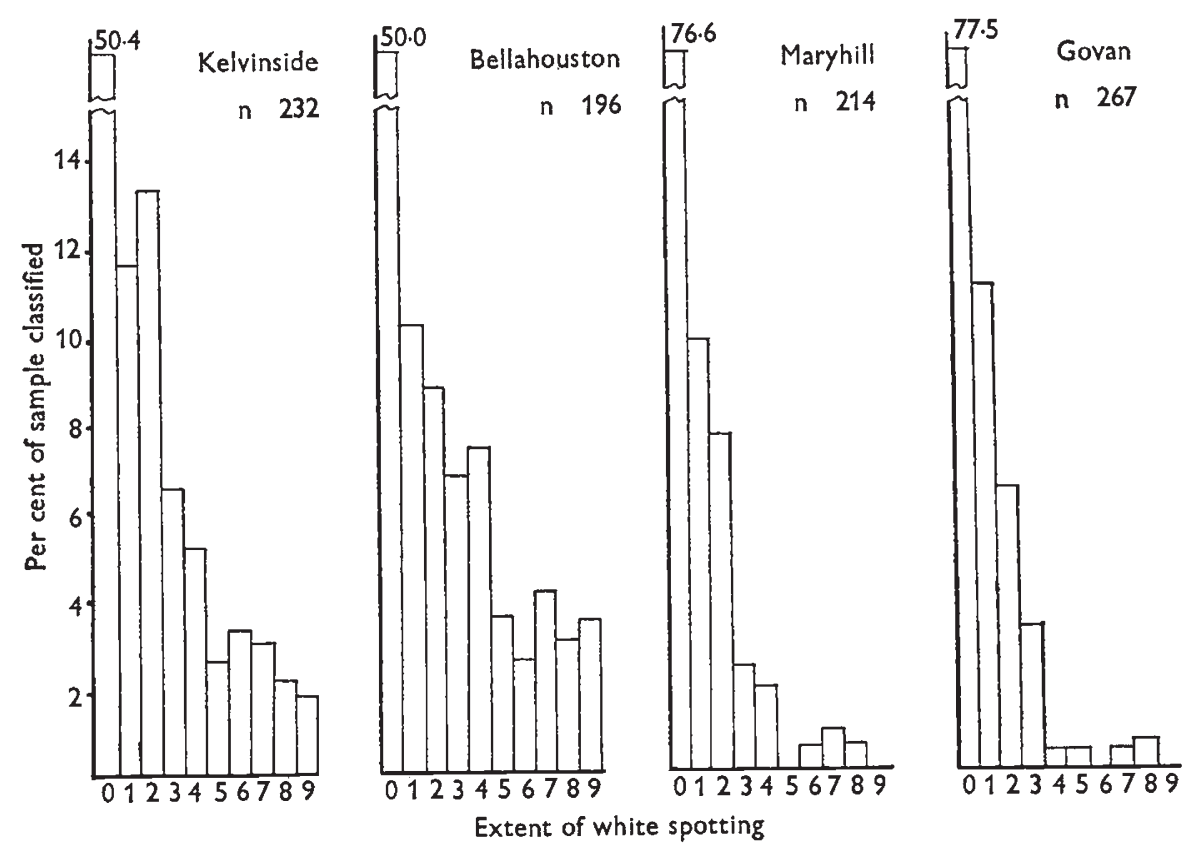

Fig. 2.-Distribution of white spotting in cats from the four regions. Extent of spotting is classified according to the scale suggested by Dreux and Legel (1973). $n$ represents the sample size.

nearest city to Glasgow to have been surveyed. The frequencies in York are thus intermediate between those in Kelvinside/Bellahouston and Maryhill/Govan samples. The samples may not be strictly comparable since the survey in York was based on animals in a welfare home and probably represents many "districts" of York. However, no wide discrepancy in gene frequency exists.

The data on distribution of white spotting show an interesting difference between the two types of area. Fig. 2 presents the percentage in each class of animals scored for this character. Kelvinside and Bellahouston exhibit similar distributions. However, when these distributions are compared with those for Maryhill and Govan it becomes apparent that not only is there an increased proportion of non-spotted animals in Maryhill and Govan, but there is also a deficiency of extensive spotting in these two areas. Dreux and Legel (1973) have suggested that more extensive spotting corresponds to the homozygote, $S S$, and the least extensive patterns to the heterozygote, $S s^{+}$. Non-spotted animals are presumed to be $s^{+} s^{+}$. Allowing 
for scoring error and variability in expression of $S$ (Dreux and Legel, 1973), the distributions shown in fig. 2 do not deviate greatly from Hardy-Weinberg expectations.

\section{Discussion}

In order to discuss the significance of these results consider table 1 . The three "populations" described in this table are: the preferred theoretical population, or a possible result of human esthetic taste; a small sample of cats actually owned by respondents to the questionnaire, described according to a specified list of phenotypes; and a large sample of cats scored in an independent study of the seven loci. Since the stated preferences apply to phenotypes, it is very difficult to translate these preferences into selection at the seven loci.

The dominant white phenotype shows the most dramatic discrepancy between "preferred" and actual population frequencies. In all samples the proportion of dominant white cats owned or observed was very low. In contrast, over 35 per cent in the sample would prefer dominant white to the alternatives listed. Such a strong selection for these cats would be expected to culminate in a frequency of $W$ well above that found in the present samples. It must be inferred that selection against $W$ greatly outweighs its preference value in all four regions studied.

The difference between the "preferred" and observed frequencies of tabby cats in the population is not great $\left(\chi^{2}<4 \cdot 72\right.$ for all areas). In all cases, the "preferred" proportion was slightly less than that observed. This may suggest a slight selection against tabby cats. Although it was not possible to accurately assess preferences for striped or blotched tabby, most respondents who preferred tabby cats regarded " tiger" cats as most desirable. This may suggest a preference for $t^{+}$.

In all areas the observed frequency of the black phenotype was well above the "preferred" level $\left(\chi^{2}>8.01\right.$ for all areas). In addition, the proportion of owned black cats in Maryhill and Govan was less than that found in the large sample of stray and owned cats. These results suggest that human preference may be a force acting to reduce the frequency of black cats.

The data for tortoiseshell show a difference between the two types of area. In Kelvinside and Bellahouston the actual proportion of tortoiseshell cats is above that preferred ( $\chi^{2}>9.60$ for both samples). In contrast, the proportion of tortoiseshell cats in Maryhill and Govan is much less and approximates the preferred value $\left(\chi^{2}<1.47\right.$ for both areas). The category for "tortoiseshell" includes "calico" cats. From table 1 no obvious pattern has emerged for $O$. In Kelvinside and Bellahouston there is little difference between the "preferred" and actual population $\left(\chi^{2}<0.67\right.$ for both cases). In Bellahouston there appear to be even more orange cats than is "preferred". In both Maryhill and Govan, human preferences may act to increase the frequency of orange cats. When questioned on the orange-tabby phenotype, most respondents preferred the striped to the blotched pattern. It was not possible to accurately assess this preference.

The purpose of including grey and cream phenotypes in the survey was to provide information on preference at the $d$ locus. In both Kelvinside and Bellahouston the "preferred" proportion of grey cats is less than that 
observed ( $\chi^{2}=4.77$ and 5.35 respectively). For cream cats the situation appears reversed ( $\chi^{2}=11.18$ and 3.50 respectively). The data for Maryhill and Govan suggest that human preference may increase the frequency of dilute phenotypes in these areas.

In both Kelvinside and Bellahouston human preference may act to reduce white spotting. However, this effect is not as great as the figures indicate. Those who preferred white spotting preferred the more extreme forms. Thus the effects of human preference would be greatest for the homozygote, SS. The fact that observed white spotting is less frequent in Maryhill and Govan suggests that selection is acting against this phenotype. There was no obvious association between preferences for white spotting and a particular phenotype. In all areas the long-hair phenotype illustrates the discrepancy between "preferred" and observed proportions. The difference is greatest in Maryhill and Govan.

It is possible to approach the data on "preferred" and owned populations in a different manner. The large preference shown by people for white cats suggests that almost any colour of cat is present in the population in excess of the percentage of owners who would like to have that colour. It is not possible to know what influence the preferences exercised by people who would like to have a white cat but cannot get one may be having on the other coat colours. If dominant white is excluded from the questionnaire data, the estimates of relative genotype frequencies shown in table 4 clearly illustrate the trends suggested by the data in table 1. All phenotypes can be classified at the $O$ locus such that black includes black, tabby and grey animals, and orange includes orange and cream animals. An estimate of the proportion of dilution was obtained by pooling grey and cream animals. This may lead to an underestimate since other dilute phenotypes were not included. The proportion of non-agouti was determined after exclusion of tortoiseshell, orange and cream cats. When the data are analysed in this way, directions of selection related to human preference become apparent. The effect of human preference would be to decrease the frequency of black and increase the frequency of orange. The frequency of dilution and long-hair will tend to be increased by human preference whereas non-agouti and white-spotting may be reduced. It is not possible to estimate the genotype frequencies for blotched tabby since preference for tabby patterns could not be accurately assessed. The results of this questionnaire confirm the observations by Dyte (1974), who suggested that there may be a preference for striped tabby, orange, and long hair amongst animals found at cat shows. The data presented in this paper do not support the idea of human preference for tortoiseshell (Dyte, 1974) and non-agouti (Searle, 1949; Robinson and Silson, 1969; Metcalfe and Turner, 1971).

Although detailed interpretation of the data is not possible, some important general observations can be made. The emergence of consistent patterns reveal the direction, but not magnitude, of selection. The methods described in this paper may not be ideal but they utilise currently available material. For example, it would be more appropriate to compare "preferred" and owned populations with stray populations. Since it is not possible to identify stray animals, an approximation has to be made by door-to-door canvass and casual observations. Obviously such a sample would be biased towards the owned and "preferred" populations but should still show characteristics of the stray population. Since the population is 


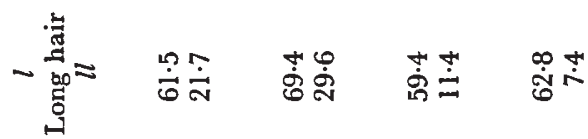

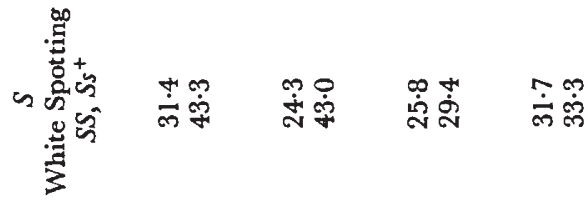

㞭

○

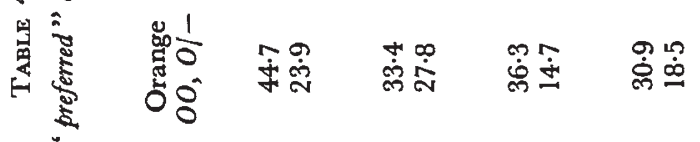

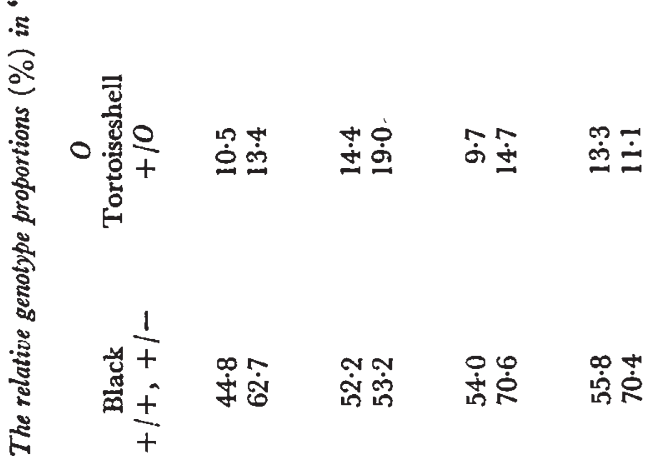

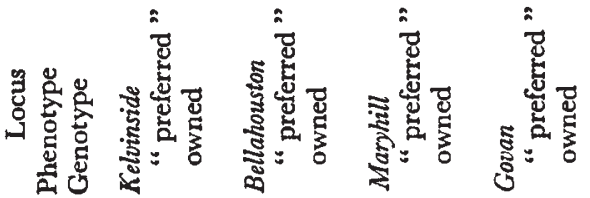


shown to be panmictic the distinction between these two groups may be small. Humans have little influence on the mate selection of cats-given that the cats are not subjected to sterilisation (Searle, 1949).

In all areas studied there was a human preference for lighter phenotypes. Where the influence of human preference is greatest (Kelvinside and Bellahouston), the cat population tends towards a lighter phenotype and where influence is least (Maryhill and Govan) the cat population tends towards a darker phenotype. This argument assumes that the influence of human preference on the cat population is related to the incidence of ownership. The assumption is also made that human preference results in selection. While these assumptions may be correct, a number of points illustrate that the role of human preferences in shifting cat gene frequencies must not be overestimated.

(1) The high risks of sterilisation effectively reduce " preferred " phenotypes in the mating population.

(2) Many of the owned cats were originally stray (possibly over 30 per cent, see Appendix (5)).

(3) The incidence of ownership is low in some areas (see Appendix (4)). Although the effective population size is not known, it is expected that the owned mating population is a small proportion of the effective population size. However, this may not be true for the more affluent areas.

(4) The owned population was different from the "preferred" (see table 1).

It is concluded that the influence of human preference is small, and where this preference is greatest, the neutering risk is also high. The effect of human preference appears greatest for $S, l, W$ and probably novel mutants. A general increase in affluence suggests that the effects of human preference are stronger now than in the past.

One cannot escape the likelihood of selection against lighter phenotypes in the Maryhill and Govan environments. Since all loci studied show a trend towards an increase in the frequency of the darker allele alternative, it is likely that this shift is due to selection rather than random events. Although there is more than one way to obtain a dark coat, the data presented here show consistent patterns towards darkness. This may suggest that one "force" is operating on one population. An explanation based on the selective neutrality of these coat colour polymorphisms, although possible, seems rather unlikely. Similarly, an explanation based on the founder effect appears inadequate. It is proposed that in the urban environment darker phenotype has a selective advantage. This advantage seems to be related to the severity of the environment. Here the environment is described in human terms. Such a situation seems analogous to "industrial (urban) melanism ". To date, the cat populations of Maryhill and Govan are the darkest to have been described in the world. Paradoxically, this process of darkening could be hastened by human selection for lighter phenotypes and associated probabilities of sterilisation.

It is not possible to identify the selective force involved in the darkening of the phenotype. The results indicate that there is an increased chance of death by accident or illness in those areas where selection appears most severe. There may be an advantage in being less conspicuous. This could 
be related to " predation" by humans-lighter phenotypes attracting more attention and thus exposure to mischief. The selection appears to be against coat colour rather than closely linked resistance to other factors since alleles at seven apparently unlinked loci behave in a similar manner, i.e. towards a darker phenotype. The role of past or present atmospheric pollution in the darkening of phenotype is probably small since Kelvinside and Maryhill, with contrasting cat populations are only a few hundred metres apart. Similarly, Govan and Bellahouston are geographically adjacent areas separated by only railway lines and a main road, and thus share a similar atmosphere.

The significant variation in gene frequency between different areas may result in sharp clines related to environment, while humans assist cat gene flow to an unknown extent. Since inhabitants of higher socioeconomic regions have greater opportunity to express their preferences, gene flow may be more significant in the direction of these areas. Such humanassisted cat gene flow may account for some of the phenotypic darkening found in lower socioeconomic regions. The fact that similar patterns have been observed in adjacent, contrasting environments in well-separated regions may suggest that the described correlation between phenotypic darkness and environment is a general phenomenon in this city. If this is the case, it is expected that there will be numerous sharp clines within the city. The cline gradients will depend on initial gene frequencies, selection related to environment, gene flow and perhaps the effects of human preference. It is possible that under some conditions, discontinuities in gene frequency rather than clines will develop. The variation in gene frequency within the city illustrates a problem in assessing gene frequency estimates in large cities. For the purpose of regional surveys it is difficult to decide what is representative for the city. Since the Maryhill/Govan type of environment tends to support a greater population of stray and unwanted animals, welfare home samples would be biased towards such areas rather than the more affluent areas.

The data presented in this paper serve a double purpose. Firstly, they may help to clarify the role of human preference in affecting urban cat populations. Although this study was undertaken in Glasgow, there is no reason to suspect that similar results would not be found in other large cities. Secondly, we have an example of selection operating on a number of polymorphic loci. This selection is made up of opposing componentsthe "artificial", human preference component favouring lighter phenotypes, and unidentified " natural " components favouring darker phenotypes.

Acknowledgments.-The author is grateful for the co-operation of people who responded to this survey, and also to Dr N. B. Todd for reading and commenting on the manuscript.

\section{REFERENGES}

CAVAlli-SFORZa, L. L., AND Bodmer, w. F. 1971. The Genetics of Human Populations, p. 795. W. H. Freeman, San Francisco.

CLARK, J. M. 1973. Gene frequencies in the domestic cats of Adelaide. Aust. F. Biol. Sci., $26,1215-1219$.

COOK, L. M. 1971. Coefficients of Natural Selection. Hutchinson, London. DREUX, PH. 1967. Gene frequencies in the cat population of Paris. F. Hered., 58, 89-92. DREUX, PH., AND LEGEL, J. C. 1973. Gene frequencies in the cat population of the Hague. 7. Hered., 64, 337-339. 
DYTE, C. E. 1974. An aspect of human selection affecting coat variation in the domestic cat. Carnivore Genetics Newsl., 2, 219-224.

METCALFE, J. A., AND TURNER, J. R. G. 1971. Gene frequencies in the domestic cats of York: evidence of selection. Heredity, 26, 259-268.

ROBinson, R. 1959. Genetics of the domestic cat. Bibliogr. Genet., 18, 273-362.

Robinson, R. 1972. Mutant gene frequencies in cats of Cyprus. Theoret. Appl. Genet., 42, 293-296.

ROBINSON, R., AND SILSON, M. 1969. Mutant gene frequencies in the cats of southern England. Theoret. Appl. Genet., 39, 326-329.

SEARLE, A. G. 1949. Gene frequencies in London's cats. F. Genet., 49, 214-220.

TODD, N. B. 1969. Cat gene frequencies in Chicago and other populations of the United States. F. Hered., 60, 273-277. 


\section{APPENDIX}

Summary of results from a questionnaire survey of human preference and cat ownership patterns

(1) What type(s) of cat do you find most attractive?

\begin{tabular}{|c|c|c|c|c|c|c|c|c|}
\hline & \multicolumn{2}{|c|}{ Kelvinside } & \multicolumn{2}{|c|}{ Bellahouston } & \multicolumn{2}{|c|}{ Maryhill } & \multicolumn{2}{|c|}{ Govan } \\
\hline & No. & $\%$ & No. & $\%$ & No. & $\%$ & No. & $\%$ \\
\hline Black & 21 & $11 \cdot 7$ & 26 & $15 \cdot 3$ & 28 & $13 \cdot 5$ & 22 & $11 \cdot 5$ \\
\hline Pure white & 74 & $41 \cdot 3$ & 59 & $34 \cdot 7$ & 83 & $40 \cdot 1$ & 71 & $37 \cdot 2$ \\
\hline Tabby & 20 & $11 \cdot 2$ & 25 & $14 \cdot 7$ & 29 & $14 \cdot 0$ & 32 & $16 \cdot 7$ \\
\hline Orange & 33 & $18 \cdot 4$ & 26 & $15 \cdot 3$ & 33 & $15 \cdot 9$ & 27 & $14 \cdot 1$ \\
\hline Grey & 6 & $3 \cdot 4$ & 7 & $4 \cdot 1$ & 10 & $4 \cdot 8$ & 13 & $6 \cdot 8$ \\
\hline Cream & 14 & $7 \cdot 8$ & 11 & $6 \cdot 5$ & 12 & $5 \cdot 8$ & 10 & $5 \cdot 2$ \\
\hline Tortoiseshell & 11 & $6 \cdot 1$ & 16 & $9 \cdot 4$ & 12 & $5 \cdot 8$ & 16 & $8 \cdot 4$ \\
\hline Coloured with white spots & 33 & $18 \cdot 4$ & 27 & $15 \cdot 9$ & 32 & $15 \cdot 5$ & 38 & $19 \cdot 9$ \\
\hline Short hair & 69 & $38 \cdot 5$ & 52 & $30 \cdot 6$ & 84 & $40 \cdot 6$ & 71 & $37 \cdot 2$ \\
\hline Long hair & 110 & $61 \cdot 5$ & 118 & $69 \cdot 4$ & 123 & $59 \cdot 4$ & 120 & $62 \cdot 8$ \\
\hline Male & 80 & $44 \cdot 7$ & 82 & $48 \cdot 2$ & 112 & $54 \cdot 1$ & 109 & $57 \cdot 1$ \\
\hline Female & 99 & $55 \cdot 3$ & 88 & $51 \cdot 8$ & 95 & $45 \cdot 9$ & 82 & $42 \cdot 9$ \\
\hline Sample size & 179 & & 170 & & 207 & & 191 & \\
\hline
\end{tabular}

(2) Do black cats bring (a) good luck? (b) bad luck? (c) doesn't matter?

Good luck

Bad luck

Doesn't matter

Sample size

Percentage superstitious

Kelvinsid
16
13
150
179

$16 \cdot 2$
Bellahouston

17
14
139
170

$\begin{array}{cc}\text { Maryhill } & \text { Govan } \\ 27 & 27 \\ 23 & 23 \\ 157 & 141 \\ 207 & 191 \\ 24 \cdot 1 & 26 \cdot 2\end{array}$

(3) Have you kept a cat in the last five years?

$\begin{array}{lcccc} & \text { Kelvinside } & \text { Bellahouston } & \text { Maryhill } & \text { Govan } \\ \text { Yes } & 51 & 69 & 32 & 23 \\ \text { Total kept } & 69 & 81 & 35 & 27 \\ \text { No } & 128 & 101 & 175 & 168 \\ \text { Sample size } & 179 & 170 & 207 & 191\end{array}$

(4) Do you keep a cat now?

$\begin{array}{lcccc} & \text { Kelvinside } & \text { Bellahouston } & \text { Maryhill } & \text { Govan } \\ \text { Yes* } & 44 & 67 & 20 & 17 \\ \text { No } & 135 & 103 & 187 & 174 \\ \text { Sample size } & 179 & 170 & 207 & 191 \\ \text { Percentage ownership* } & 24 \cdot 6 & 39 \cdot 4 & 9 \cdot 7 & 8 \cdot 9\end{array}$

* Includes ownership of more than one animal. 
(5) What was the origin of these animals?

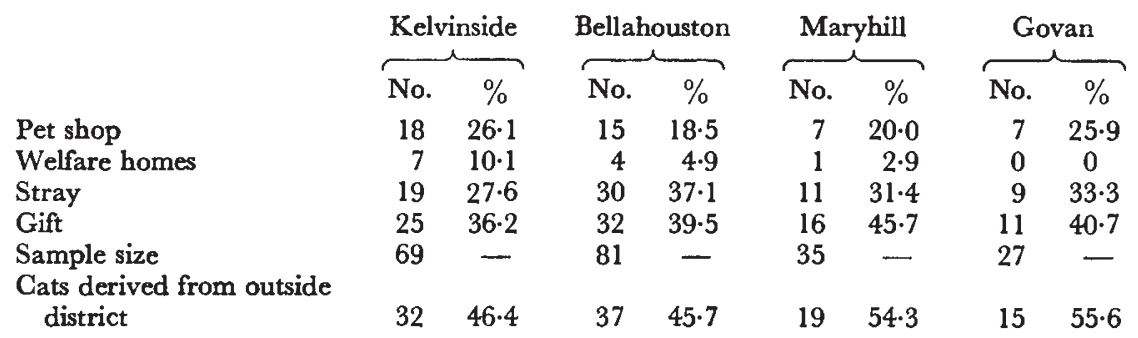

(6) Did children influence the choice of cat you have kept?

$\begin{array}{lcccc} & \text { Kelvinside } & \text { Bellahouston } & \text { Maryhill } & \text { Govan } \\ \text { Yes } & 21 & 29 & 7 & 6 \\ \text { No } & 48 & 52 & 28 & 21 \\ \text { Total } & 69 & 81 & 35 & 27\end{array}$

(7) Were the cats you have kept (a) male? (b) female? (c) neuter male? (d) neuter female?

Male

Female

Neuter male

Neuter female

Sample size

$\begin{array}{cccc}\text { Kelvinside } & \text { Bellahouston } & \text { Maryhill } & \text { Govan } \\ 14 & 23 & 13 & 11 \\ 17 & 15 & 10 & 8 \\ 13 & 21 & 7 & 5 \\ 25 & 22 & 5 & 3 \\ 69 & 81 & 35 & 27\end{array}$

(8) Describe the cause of death of any of your cats in the past five years.

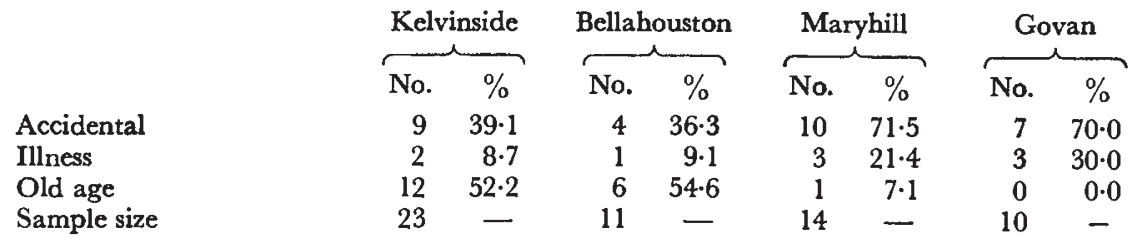

(9) Describe the cats you have kept in the last five years.

\begin{tabular}{|c|c|c|c|c|c|c|c|c|}
\hline & \multicolumn{2}{|c|}{ Kelvinside } & \multicolumn{2}{|c|}{ Bellahouston } & \multicolumn{2}{|c|}{ Maryhill } & \multicolumn{2}{|c|}{ Govan } \\
\hline & No. & $\%$ & No. & $\%$ & No. & $\%$ & No. & $\%$ \\
\hline Black & 24 & $34 \cdot 8$ & 22 & $27 \cdot 2$ & 15 & $42 \cdot 9$ & 9 & $33 \cdot 3$ \\
\hline Pure white & 2 & $2 \cdot 9$ & 2 & $2 \cdot 5$ & 1 & $2 \cdot 9$ & 0 & 0 \\
\hline Tabby & 14 & $20 \cdot 3$ & 15 & $18 \cdot 5$ & 8 & $22 \cdot 9$ & 9 & $33 \cdot 3$ \\
\hline Orange & 15 & $21 \cdot 7$ & 19 & $23 \cdot 5$ & 5 & $14 \cdot 3$ & 5 & $18 \cdot 5$ \\
\hline Grey & 4 & $5 \cdot 8$ & 5 & $6 \cdot 2$ & 1 & $2 \cdot 9$ & 1 & $3 \cdot 7$ \\
\hline Cream & 1 & 1.4 & 3 & $3 \cdot 7$ & 0 & $0 \cdot 0$ & 0 & $0 \cdot 0$ \\
\hline Tortoiseshell & 9 & $13 \cdot 0$ & 15 & $18 \cdot 5$ & 5 & $14 \cdot 3$ & 3 & $11 \cdot 1$ \\
\hline Coloured with white spots & 29 & $42 \cdot 0$ & 34 & $42 \cdot 0$ & 10 & $28 \cdot 6$ & 9 & $33 \cdot 3$ \\
\hline Long hair & 15 & $21 \cdot 7$ & 24 & $29 \cdot 6$ & 4 & $11 \cdot 4$ & 2 & $7 \cdot 4$ \\
\hline Short hair & 54 & $78 \cdot 3$ & 57 & $70 \cdot 4$ & 31 & $88 \cdot 6$ & 25 & $92 \cdot 6$ \\
\hline Sample size & 69 & & 81 & & 35 & & 27 & \\
\hline
\end{tabular}

